\title{
Brugada syndrome unmasked by ischemia needs full risk evaluation: author's reply
}

\author{
Pankaj Garg • Thomas Nelson • Jonathan Sahu • \\ Paul Sheridan
}

Received: 29 October 2014/ Accepted: 5 November 2014/Published online: 20 November 2014

(C) SIMI 2014

\section{Dear Editor,}

We are much obliged to Gottschalk et al. [1] for the clarification of the morphological classification of Brugada syndrome $(\mathrm{BrS})$. As suggested initially, we were describing Brugada phenocopy (BrP) with myocardial ischemia [2]. This was ruled out after a positive Ajmaline test for BrS.

As pointed out by Gottschalk et al., we initially felt that the co-existence of coronary artery disease and $\mathrm{BrS}$ places the patient at high risk for adverse cardiac events. However, after the revascularisation of the right coronary artery (RCA), the patient had a treadmill exercise test (ETT), and a one-week monitor evaluation, both of these tests did not show any significant dysrhythmias or ischemia. During his clinic visit, details of history were taken, and no previous family history of sudden death was identified. Also, it was noted that there was no clinical history of syncope. As per the latest HRS/EHRA/APHRS consensus statement, we offered him the following therapeutic interventions: lifestyle changes advice involving avoidance of drugs that may induce or aggravate ST segment elevation in right precordial leads (Brugadadrugs.org), avoidance of excessive alcohol intake, and immediate treatment of fever with antipyretic drugs [3]. The option of implantable cardiac defibrillator (ICD) therapy was raised and discussed, however, as he remained a completely asymptomatic BrS patient with no family history of sudden cardiac death, a multidisciplinary team including the patient decided against it. He remains under our active vigilant follow-up in the inherited cardiac condition clinic where detailed history is revisited, and lifestyles modifications are reemphasised.

Conflict of interest None.

\section{References}

1. Gottschalk BH, Anselm DD, Baranchuk A (2014) Brugada syndrome unmasked by ischemia needs full risk evaluation. Intern Emerg Med. doi:10.1007/s11739-014-1145-9

2. Garg P, Nelson T, Sahu J, Sheridan P (2014) Is this ST-elevation because of myocardial ischaemia or a Brugada pattern? An interesting case review. Intern Emerg Med. doi:10.1007/s11739014-1127-y

3. Priori SG, Wilde AA, Horie M, Cho Y, Behr ER, Berul C et al (2013) HRS/EHRA/APHRS expert consensus statement on the diagnosis and management of patients with inherited primary arrhythmia syndromes: document endorsed by HRS, EHRA, and APHRS in May 2013 and by ACCF, AHA, PACES, and AEPC in June 2013. Heart Rhythm 10(12):1932-1963
P. Garg $(\bowtie) \cdot$ T. Nelson · J. Sahu · P. Sheridan Sheffield Teaching Hospitals NHS Foundation Trust, Sheffield, UK

e-mail: pankajvic@gmail.com 